\title{
Task-order coordination in dual-task performance and the lateral pre- frontal cortex: an event-related fMRI study
}

Received: 2 July 2004 / Accepted: 15 April 2005 / Published online: 2 September 2005

(C) Springer-Verlag 2005

\begin{abstract}
A crucial demand in dual tasks suffering from a capacity limited processing mechanism is task-order scheduling, i.e. the control of the order in which the two component tasks are processed by this limited processing mechanism. The present study aims to test whether the lateral prefrontal cortex (LPFC) is associated with this demand. For this, 15 participants performed a psychological refractory paradigm (PRP) type dual task in an event-related functional magnetic resonance (fMRI) experiment. In detail, two choice reaction tasks, a visual (response with right hand) and an auditory (response with left hand), were presented with a temporal offset of $200 \mathrm{~ms}$, while the participants were required to respond to the tasks in the order of their presentation. Importantly, the presentation order of the tasks changed randomly. Based on previous evidence, we argue that trials in which the present task order changed as compared to the previous trial (differentorder trials) impose higher demands on task coordination than same-order trials do. The analyses showed that cortical areas along the posterior part of the left inferior frontal sulcus as well as the right posterior middle
\end{abstract}

A. J. Szameitat $(\varangle) \cdot$ D. Y. von Cramon

Max Planck Institute for Human Cognitive and Brain Sciences, Leipzig, Germany

J. Lepsien

Department of Psychology, University of Oxford, Oxford, UK

A. Sterr

Department of Psychology, University of Surrey, Guildford, UK

T. Schubert

Department of Psychology, Humboldt University,

Berlin, Germany

Present address: A. J. Szameitat

Department of Psychology, School of Human Sciences, University of Surrey, Guildford, Surrey GU2 7XH,

United Kingdom

E-mail: a.szameitat@surrey.ac.uk

Tel.: + 44-1483-682881

Fax: + 44-1483-682914 frontal gyrus were more strongly activated in differentorder than in same-order trials, thus supporting the conclusion that one function of the LPFC for dual-task performance is the temporal coordination of two tasks. Furthermore, it is discussed that the present findings favour the active scheduling over the passive queuing hypothesis of dual-task processing.

\section{Introduction}

Performing dual tasks usually results in performance decrements as compared to single-task performance (for a review, see e.g. Pashler, 1994). Such decrements, evident as prolonged reaction times and/or higher error rates, are indicative of additional cognitive demands in the dual-task situation as compared to the isolated performance of the single tasks (Braun, 1998; De Jong, 1995; Duncan, 1979; Logan \& Gordon, 2001; Luria \& Meiran, 2003; Meyer \& Kieras, 1997). Surprisingly, the question of how the brain handles these additional demands is still largely open. This question is highly important, because its answer allows deeper understanding of the fundamental brain mechanisms regulating goal-directed behaviour in complex situations with contradictory and interfering information.

In recent years, a number of imaging studies aimed at identifying the neuroanatomical correlates of the additional cognitive demands in dual-task performance. The findings of these previous imaging studies suggested a specific role of the lateral prefrontal cortex (LPFC) for dual-task processing (D'Esposito, Detre, Alsop, Shin, Atlas, \& Grossman, 1995; Goldberg et al., 1998; Schubert \& Szameitat, 2003; but see Jiang, Saxe, \& Kanwisher, 2004). Specifically, these studies compared the fMRI activation in dual-task blocks with the activation when participants performed the component tasks in single-task blocks, thereby employing the method of cognitive subtraction (Friston, Price, Fletcher, Moore, 
Frackowiak, \& Dolan, 1996). The finding of an overadditive activation in prefrontal regions was interpreted as being related to additional cognitive demands present in the dual task, as compared to the summed demands of both single tasks. However, while these studies are highly valuable for the localisation of the neuroanatomical correlates of dual-task performance, they do not allow specifying which specific processes are in particular associated with the dual-task related LPFC activation. This is, because the results of a gross comparison between dual-task and single-task blocks reflect the influence of numerous dual-task related demands. Note, for example, that the amount of the working memory load, the need to divide attention, as well as the need to coordinate task processing may differ between dual-task and single-task blocks and, accordingly, may be responsible for the observed activation difference (cf. Jiang et al., 2004). The aim of the present study is, therefore, to specify the processes which are related to the activation of the lateral prefrontal cortex during dual-task coordination.

The starting point of the present study are findings of a previous study of ours (Szameitat, Schubert, Müller \& von Cramon, 2002). In that study, we used the method of parametric manipulation ${ }^{1}$ in order to show that the LPFC is in particular associated with the coordination of the temporal order in which the component tasks are processed (hereafter called "task-order scheduling"). According to a number of authors, the need for taskorder scheduling emerges in situations where two processing stages or streams compete for access to a capacity limited processing stage, like a bottleneck mechanism in dual tasks of the psychological refractory period (PRP) type (Fig. 1, De Jong, 1995; Luria \& Meiran, 2003; Umiltà, Nicoletti, Simion, Tagliabue, \& Bagnara, 1992; but see Pashler, 1994) ${ }^{2}$. The authors suggested that this competition is resolved by control mechanisms coordinating, i.e. planning and monitoring, the order in which the tasks are processed. In order to localise the function of task-order scheduling, we compared two dual-task conditions with different demands on task-order scheduling, namely dual-task blocks with a fixed presentation order of the two component tasks and blocks with a random presentation order. Since

\footnotetext{
${ }^{1}$ According to the method of parametric manipulation the neuroanatomical correlate of a specific process can be localised by manipulating the difficulty of that process. Those brain regions, which change their activation in relation to the difficulty manipulation are assumed to be the neural substrate associated with the manipulated process (see e.g. Braver, Cohen, Nystrom, Jonides, Smith, \& Noll, 1997).

${ }^{2}$ Some authors question the existence of a structural capacity limitation but instead suggest that participants strategically introduce partial serial processing of the tasks, e.g. because it may be faster and/or may help to avoid errors (Logan \& Gordon, 2001; Meyer \& Kieras, 1997). However, as soon as there is serial processing, irrespective of its structural or strategic nature, the question in which order the tasks should be processed arises. Accordingly, task-order scheduling is required under both assumptions, and the current findings apply to both of them.
}

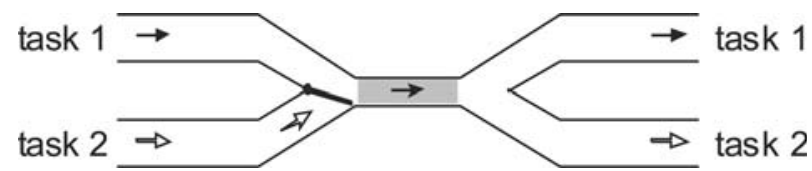

Fig. 1 Illustration of a capacity limited processing stage, i.e. a bottleneck (shaded grey). Task-order scheduling is required because both tasks compete for the processing by the bottleneck

participants responded to the tasks in the order of their appearance, task processing had to be rearranged in random-order blocks whenever the presentation order changed (De Jong, 1995; Luria \& Meiran, 2003). Therefore, task-order scheduling was more demanding in random-order as compared to fixed-order blocks, in which the same scheduling strategy could be applied throughout a block. As a result, the LPFC was more strongly activated in random- than in fixed-order blocks, which led to our conclusion that the LPFC is associated with task-order scheduling in dual-task situations. The findings of this experiment extended previous knowledge in two aspects: Firstly, they replicated and confirmed the finding of cognitive subtraction studies that the LPFC is associated with dual-task performance with the method of parametric manipulation. But more importantly, they established a way of specifying the function of the LPFC for dual-task performance.

However, the findings of Szameitat et al. (2002) are not completely conclusive in indicating an association of the LPFC with task-order scheduling. This is, because in that study the use of a block design may have resulted in some further confounding influences, which may hamper the final interpretation of the findings. For instance, in random-order blocks, participants do not know which task is presented first, while they do so in fixed-order blocks. Accordingly, the demands to divide attention between both tasks might have been different in both dual-task conditions. Thus, differences in divided attention may have caused the activation difference between random-order and fixed-order blocks, just like differences in task-order scheduling demands as proposed in Szameitat et al. As a further caveat, the low temporal resolution of the used fMRI block design prevents a fine grained analysis of those processes, which are finally related to the observed fMRI activation. Note, that a block design implicates a summation of the activation across the whole block, and, therefore, does not allow the analysis of processes on the level of single trials.

The present study aimed at characterising the role of lateral-prefrontal cortices for dual-task performance in more detail by avoiding the above mentioned possible methodological shortcomings. For this, we employed, to our knowledge as the first imaging study of dual-task performance, an event-related fMRI design (Rosen, Buckner, \& Dale, 1998). This allows a more elaborate manipulation of task-order scheduling as compared to Szameitat et al. (2002). In particular, the present experimental manipulation is now based on a trial-wise 
modulation of task-order scheduling as proposed by De Jong (1995) and Luria and Meiran (2003). These authors have shown that participants are able to prepare the processing order of the two tasks of a dual-task situation before the beginning of a trial. In particular, it has been shown that participants prepare to process the tasks in the actual trial in the same order as they have been processed in the previous trial. Therefore, in trials with the same order (same-order trials) as in the preceding trial, participants are prepared to process the correct task first, and the task-order scheduling demands are rather low. On the contrary, if the presentation order changes between trials (different-order trials), participants are prepared to process the wrong task as the first task. In that case participants have to overcome the preparation of the wrong task by additional cognitive processing related to the re-organisation of task processing, for instance by activating the correct task and/ or discarding the wrong task (De Jong, 1995; Luria \& Meiran, 2003). Accordingly, in different-order trials, the demands on task-order scheduling increase as compared to same-order trials. Note, that same- and different-order trials are basically identical, they only differ with respect to the presentation order of the tasks in the preceding trial. Thus, different to Szameitat et al. (2002), the two conditions now differ merely with respect to task-order scheduling demands, but not with respect to demands on divided attention. Because the upcoming task order is unpredictable in the present study, participants have to divide their attention in same- as well as in different-order trials. Thus, if the lateral-prefrontal cortex is associated with task-order scheduling in dualtask situations, then it should be activated more strongly in different-order than in same-order trials.

\section{Methods}

\section{Subjects}

Fifteen neurologically healthy participants (eight women) took part in the experiment, each having given prior informed consent according to the Max Planck Institute guidelines. The study was approved by the local ethics review board at the University of Leipzig, Germany. All participants were right handed as assessed by the Edinburgh Inventory (Oldfield, 1971) and had normal or corrected to normal vision. The age ranged from 22 to 29 years (average 24 years).
Stimuli

Participants performed a PRP-type dual task, consisting of two 3-choice reaction tasks, one auditory and one visual (Fig. 2). A dual-task trial started with a green screen with three horizontally arranged crosses (each $0.38^{\circ} \times 0.38^{\circ}$ ) which were presented for $1,100 \mathrm{~ms}$. The middle cross was located at the centre of the screen and served as fixation cross. The two other crosses were located horizontally to the left and right, each with a gap of $2^{\circ}$. After the presentation the screen was cleared and the target stimuli were presented with a stimulus onset asynchrony (SOA) of $200 \mathrm{~ms}$. Prior testing revealed this SOA as appropriate for convenient order detection (cf. De Jong, 1995; Szameitat et al., 2002). The target stimulus of the visual task consisted of a black square $\left(1.6^{\circ} \times\right.$ $1.6^{\circ}$ ) presented for $200 \mathrm{~ms}$ at one of the three locations indicated by the crosses of the fixation period. The target stimulus of the auditory task was a $200 \mathrm{~ms}$ lasting tone with a frequency of either 200,600 , or $1,800 \mathrm{~Hz}$. The presentation order of target stimuli was changed pseudorandomly, while it was guaranteed that in half of the trials, the auditory stimulus was presented first and in the other half the visual stimulus, and that half of the trials were different-order trials and the other half sameorder trials. Due to the SOA of $200 \mathrm{~ms}$ the presentation of both targets lasted $400 \mathrm{~ms}$. After the presentation of both targets, the screen was cleared for a response period of $2,100 \mathrm{~ms}$. Participants were allowed to respond from the onset of the first target until the end of the response period, i.e. they had to respond within 2,500 $\mathrm{ms}$ after the first target appeared. Participants responded to the left visual square with their right index finger, to the middle square with their right middle finger, and to the right square with their right ring finger. The low tone $(200 \mathrm{~Hz})$ required a reaction with the left ring finger, the middle tone $(600 \mathrm{~Hz})$ with the left middle finger and the high tone $(1,800 \mathrm{~Hz})$ with the left index finger. After the response period, either a blank screen or a visual error feedback was presented for $300 \mathrm{~ms}$. Altogether, one trial lasted 3,900 ms.

The inter-trial-interval (ITI) was randomly either $2,100,4,100$ or $6,100 \mathrm{~ms}$ (on average $4,100 \mathrm{~ms}$ ). This jittering was implemented for better estimation of the hemodynamic response (Burock, Buckner, Woldorff, Rosen, \& Dale, 1998; Friston, Zarahn, Josephs, Henson, \& Dale, 1999). During the ITI only the central fixation cross was presented and had to be fixated by the participants.
Fig. 2 Timecourse of a dualtask trial, in which the auditory task is presented first. The grey bar denotes the presentation of the tone. The inter-trial-interval was either $2,100,4,100$ or $6,100 \mathrm{~ms}$

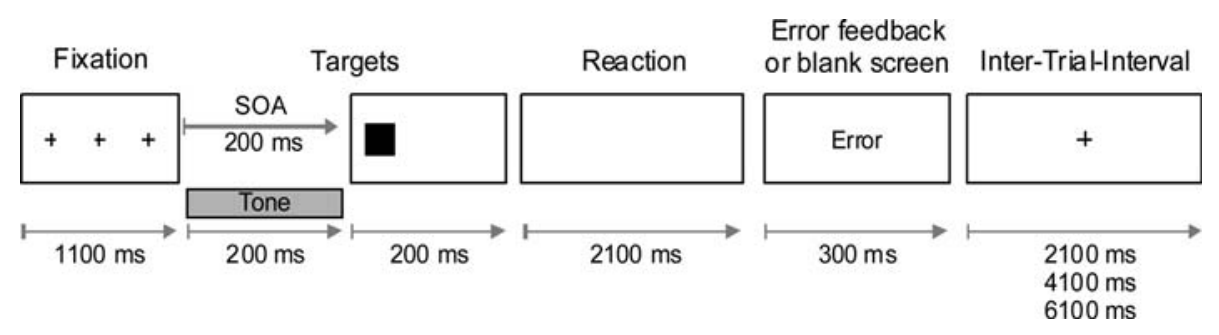


To estimate the baseline signal we additionally presented so-called null events (Friston et al., 1999; Josephs $\&$ Henson, 1999). These null events were pseudo-randomly included in the distribution of dual-task trials and equalled them concerning timing, i.e. they lasted 3,900 ms plus the jittering ITI. During a null event, only the central fixation cross was presented. Accordingly, null events were not obvious to the participants but were only recognisable by the longer duration between two trials. Participants were informed about the varying gaps between trials and instructed to fixate the central cross during such events.

\section{Design}

An event-related design was used. The experiment consisted of 72 same-order trials, 72 different-order trials, and 36 null events. Because it was unknown whether the preparatory state of the task order was still present after the presentation of a null event, task trials following null events were discarded from the analysis. Since null events could repeat, this resulted in the exclusion of 24 task trials, leaving 60 same-order and 60 different-order trials for the analysis. Thus, the experiment consisted of 180 trials and lasted $24 \mathrm{~min}, 13 \mathrm{~s}$.

To increase the temporal resolution of the measured BOLD signal, we used the method of oversampling (Josephs, Turner, \& Friston, 1997; Miezin, Maccotta, Ollinger, Petersen, \& Buckner, 2000). For this purpose, half of the trials were delayed by $500 \mathrm{~ms}$ relative to the onset of image acquisition (TR $=1 \mathrm{~s}$ ). To account for this shift, the ITI was shortened accordingly by $500 \mathrm{~ms}$, resulting in additional ITIs of $1.6,3.6$ or $5.6 \mathrm{~s}$. Thus, the oversampling method also resulted in a further jittering of the ITI (six different ITIs in total).

Due to the sluggishness of the hemodynamic response (Rosen et al., 1998), event-related designs with rather short ITIs, as in the present experiment, suffer from transitional effects of one trial to the next. To control for such effects, the transition probabilities, repetitions and the distribution of the conditions across the time course of the experiment were balanced in the best possible way. This balancing was done for the factors oversampling, jittering, auditory stimulus, visual stimulus, the combination of auditory and visual stimulus, and the experimental conditions of same-order, different-order, and null event. Each participant received a different stimulus protocol.

One to three days before the fMRI measurement, participants practiced the task outside the scanner for approximately $40 \mathrm{~min}$.

\section{Scanning procedure}

The experiment was performed at the Max Planck Institute for Human Cognitive and Brain Sciences in Leipzig, Germany (former Max Planck Institute of
Cognitive Neuroscience). Imaging was carried out with a 3T scanner (Medspec 30/100, Bruker, Ettlingen, Germany) equipped with a standard birdcage head coil. Participants were supine on the scanner bed, and cushions were used to reduce head motion. Fourteen axial slices $(19.2 \mathrm{~cm}$ FOV, $64 \times 64$ matrix, $5 \mathrm{~mm}$ thickness, $2 \mathrm{~mm}$ spacing), parallel to the AC-PC plane and covering the whole brain were acquired using a single shot, gradient recalled EPI sequence (TR $1 \mathrm{~s}$, TE $30 \mathrm{~ms}, 90^{\circ}$ flip angle) sensitive to BOLD contrast. One functional run with 1,453 volumes was administered, with each volume sampling all 14 slices. Prior to the functional run, 14 anatomical MDEFT slices and 14 EPI-T1 slices were acquired. In a separate session, high-resolution whole-brain images were acquired from each participant using a T1-weighted three-dimensional segmented MDEFT sequence. These images were linearly rotated and translated into the stereotactic space of Talairach and Tournoux (1988).

\section{Data analysis}

\section{Preprocessing}

The fMRI data were analyzed using the software package LIPSIA (Lohmann, Müller, Bosch, Mentzel, Hessler, Chen, et al., 2001). First, the functional data were pre-processed. For this purpose, the first seven timesteps were removed and a slicewise movement correction in the transverse direction was applied (Lohmann et al., 2001). A Gaussian spatial filter (FWHM $6.35 \mathrm{~mm}$ ) was used for smoothing. The temporal offset between acquisition times of different slices acquired in one volume were corrected using sinc interpolation.

After pre-processing, the functional and anatomical data were co-registered: First, the MDEFT and EPI-T1 slices geometrically aligned with the functional slices were linearly co-registered with the high-resolution 3-D reference T1 data set of each participant. Rotational and translational parameters computed for this registration were stored in individual transformation matrices. Second, each transformation matrix was transformed into a standard brain size (Talairach \& Tournoux, 1988) by linear scaling. Finally, these normalised transformation matrices were applied to the individual fMRI data. Next, the functional data were spatially re-scaled to a resolution of $3 \times 3 \times 3 \mathrm{~mm}^{3}$ using trilinear interpolation. After this linear co-registration and re-scaling, functional and anatomical data were co-registered nonlinearly: First, the anatomical image of the subject which showed the least deviation from the averaged image of all individual anatomies was taken as reference image. Second, each anatomical image was morphed in a nonlinear fashion onto this standard image (Thirion, 1998) and the individual transformation matrices were stored. Finally, these transformation matrices were applied to the individual fMRI data. 
Statistics

Statistical analysis was based on a voxelwise least squares estimation using the general linear model for serially auto-correlated observations (Friston, Holmes, Worsley, Poline, Frith, \& Frackowiak, 1995). A hemodynamic response function with two parameters (amplitude as parameter of interest and time derivative as covariate) and a response delay of $6 \mathrm{~s}$ was used to generate the design matrix. Low frequency signal drifts were controlled by applying a temporal highpass filter with a cutoff frequency of $0.014 \mathrm{~Hz}$. In addition, the design matrix and the functional data were linearly smoothed with a 4-s FWHM Gaussian kernel. For the second-level group analysis, individual contrast images were calculated by subtracting the corresponding parameter estimates from each other. In a second step, a voxel-wise one-sample $t$ test was calculated based on all individual contrast images (random effect model), resulting in a $t$-map reflecting the group statistic. Finally, the $t$-map was transformed into a $z$-map. To account for the possibility of false positives (Type-I error) we used a rather high threshold for the $z$-map $(z>3.3, P<$ 0.0005 , one-tailed, uncorrected) and an extent threshold of 7 voxel (i.e., $441 \mathrm{~mm}^{3}$ ).

\section{Results}

\section{Behavioural data}

For the following analyses, we used one-sample paired $t$ tests with the significance level calculated two-tailed. The error analysis was performed with arcsine-transformed relative error frequencies.

Different-order trials were more difficult than sameorder trials (Fig. 3). This is shown by an analysis of the reaction times (RTs), yielding increased RTs in differentorder compared to same-order trials. For the first task, this difference was $66 \mathrm{~ms}, t(14)=7.27, P<0.001$, and for the second task $72 \mathrm{~ms}, t(14)=7.54, P<0.001$. The RT increases for the first and second task did not differ, $t(14)=0.86, P=0.406$, indicating that the order manipulation had an additive effect on the RTs in both tasks. The same pattern of results was evident in the error rates, i.e. different-order trials showed higher error rates than same-order trials. For the first task, this difference was $5.3 \%, t(14)=4.069, P<0.01$, and for the second task $6.4 \%, t(14)=4.046, P<0.01$ The increase in errors rates tended to be higher in the second task as compared to the first task, $t(14)=2.06, P>0.06$. Because in a single trial, errors could occur either individually in the first or second task or concurrently in both tasks, the overall rate of erroneous trials is not evident from the just mentioned figures. The overall error rate (Fig. 3, bars) was higher by $8 \%$ in differentorder trials than in same-order trials, $t(14)=5.56, P<$ 0.001 .

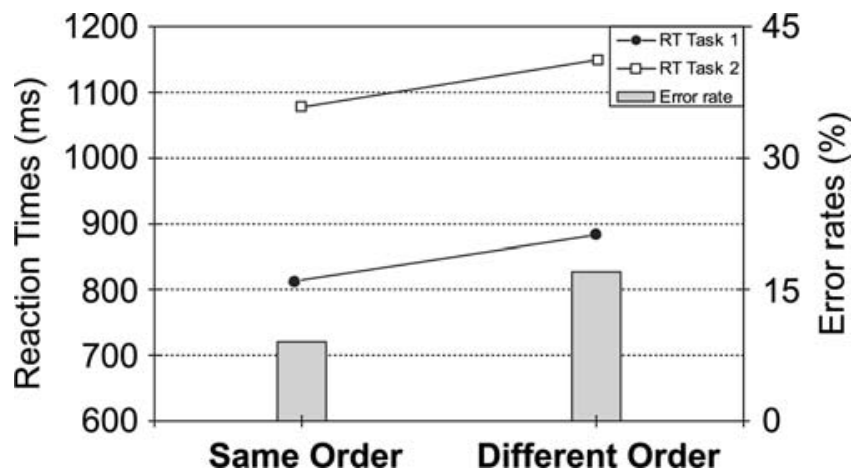

Fig. 3 Behavioural Data. Left axis and lines denote reaction times of the first (RT1, filled symbols) and second task (RT2, open symbols). Right axis and bars denote the error rates. Different-order trials showed significantly higher reaction times and error rates than same-order trials

\section{FMRI data}

In the first step, we determined cortical areas associated with task-order scheduling by calculating the contrast (different-order trials) - (same-order trials), (see Table 1 and Fig. 4, upper row). This contrast revealed a large activation in the right LPFC with a peak in the middle frontal gyrus (MFG), which extended into the inferior frontal sulcus (IFS) and the junction area of the IFS and the precentral sulcus (inferior frontal junction, IFJ). In the left LPFC, we observed a more circumscribed activation along the posterior part of IFS, which extended into the IFJ as well. Besides prefrontal activations, the comparison of different- and same-order trials revealed cortical areas exclusively in the right hemisphere. There was one focus of activation in the superior parietal lobe, along the posterior part of the horizontal and the descending segment of the intraparietal sulcus (IPS). In addition, we observed an inferior parietal activation in the supra-marginal gyrus. Finally, we found two further activated areas, one in the posterior inferior temporal gyrus and one in the medial occipital gyrus.

In the second step, we performed a regions-of-interest (ROI) analysis for the peak voxel in the left IFS $(-35,5$, 27) and the right MFG (31, 5, 41), (Fig. 4, lower row). For this, we first calculated an average signal course for a period of $11 \mathrm{~s}$ after trial onset for each condition and participant. Then, we determined individually for each participant and ROI, the percent signal change (PSC) of the conditions different-order and same-order trials by relating the signal courses of these conditions to the signal course of the null events. Thus, the PSC was calculated individually for each timestep. To test for a significant difference between different-order and sameorder trials, we averaged the PSC values across the main part of the hemodynamic response, i.e. from 4 to $8 \mathrm{~s}$ (both inclusive), and calculated an one-sample paired $t$ test (two-sided). In both prefrontal areas, different-order trials exhibited a significantly higher PSC than sameorder trials, $t(14)=3.41, P<0.01$, for the left IFS and $t(14)=3.13, P<0.01$, for the right MFG. This in- 
Table 1 Stereotactic coordinates (Talairach \& Tournoux, 1988), anatomical locations, and $z$ scores of peak activation in the comparison (different-order $>$ same-order)

\begin{tabular}{|c|c|c|c|c|c|c|c|c|}
\hline Cortical Area & Hemi & $\mathrm{BA}$ & $\mathrm{x}$ & $\mathrm{y}$ & $\mathrm{z}$ & Voxel & $\mathrm{mm}^{3}$ & $z$ \\
\hline \multicolumn{9}{|l|}{ Frontal } \\
\hline Inf frontal $\mathrm{S}$ & $\mathrm{L}$ & $9 / 44 / 6$ & -35 & 5 & 27 & 7 & 458 & 3.55 \\
\hline Middle frontal $\mathrm{G}$ & $\mathrm{R}$ & $9 / 6$ & 31 & 5 & 41 & 24 & 1547 & 4.42 \\
\hline \multicolumn{9}{|l|}{ Other } \\
\hline Supramarginal G & $\mathrm{R}$ & 40 & 59 & -52 & 27 & 14 & 917 & 3.54 \\
\hline Inf temporal G & $\mathrm{R}$ & 37 & 43 & -58 & -3 & 7 & 449 & 3.78 \\
\hline Intraparietal S & $\mathrm{R}$ & 7 & 31 & -76 & 44 & 10 & 667 & 3.67 \\
\hline Medial occipital G & $\mathrm{R}$ & 19 & 41 & -79 & 18 & 15 & 985 & 4.32 \\
\hline
\end{tabular}

Volume of the activated areas is given in the number of raw data voxels (Voxel) and in volume $\left(\mathrm{mm}^{3}\right)$. Abbreviations: Hemi $=$ Hemisphere ( $L=$ left, $R=$ right); $B A=$ Brodmann's area; $G=$ gyrus; $S=$ sulcus; $I n f=$ inferior; $\operatorname{Sup}=$ superior

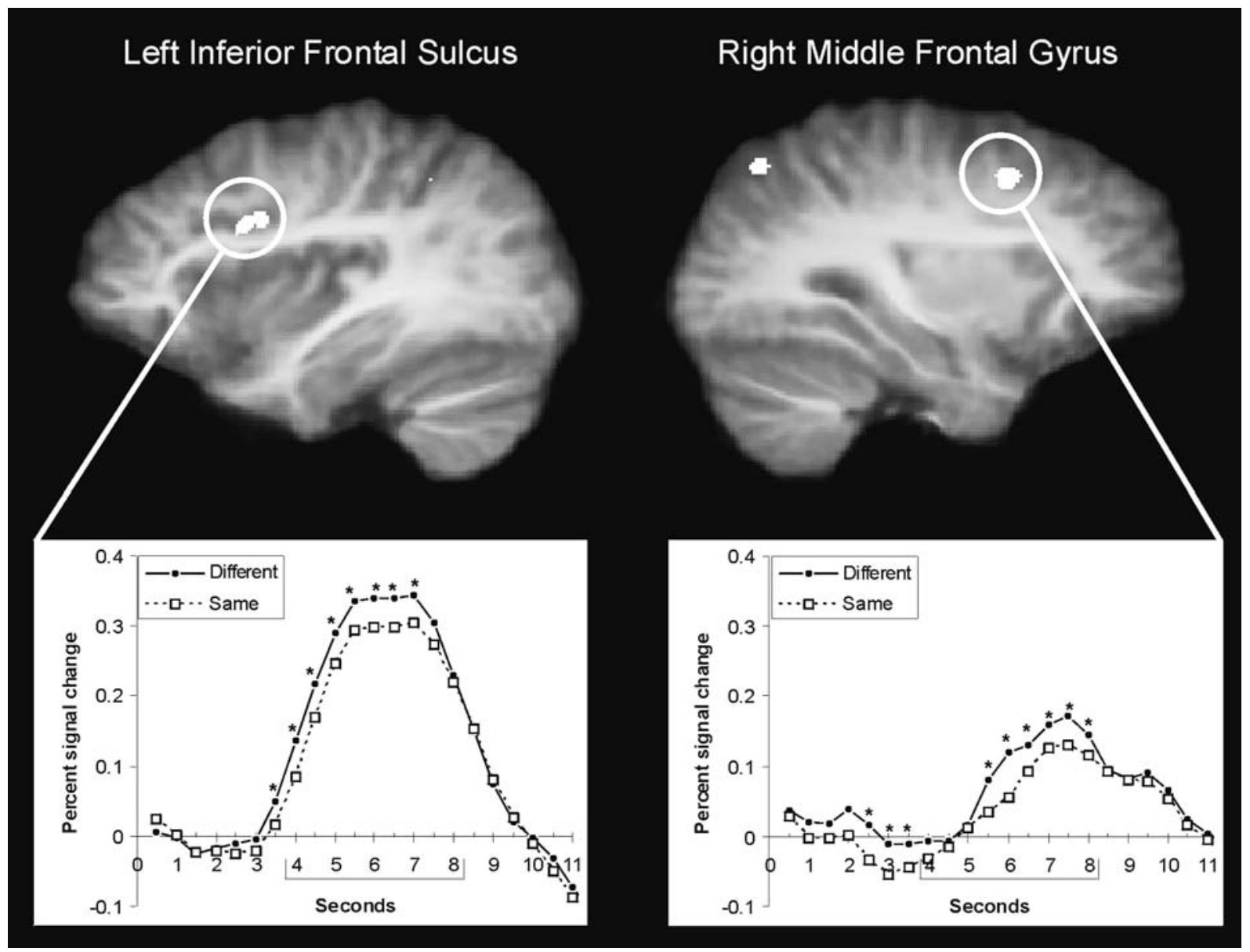

Fig. 4 Upper row. Activation maps for the comparison (differentorder $>$ same-order), thresholded at $z>3.3$. Cortical areas (encircled) along the left posterior part of the IFS and in the right posterior MFG are more strongly activated in different-order than in same-order trials. Maps are projected onto saggital images of the non-linearly averaged individual anatomies at $x=-35$ (left image) and $x=+31$ (right image) according to Talairach \& Tournoux (1988). Lower row. ROI analyses of the voxel of peak activation in

dicates, that different-order trials result in higher activity in lateral prefrontal areas than same-order trials. This holds true for an analysis based on parameter estimates the left IFS and right MFG. When averaged across seconds 4-8 (binned area of $x$-axis) different-order trials exhibit a significant higher percent signal change (PSC) than same-order trials. Asterisks denote a significant difference between order-change and order-repetition trials at individual timepoints $(P<0.05$, onesided). Percent signal change was calculated relative to null-events individually for each ROI and timepoint

according to the general linear model, as well as one that is based on the PSC calculated directly from the raw data. 
As illustrated in Fig. 4 (lower row), all areas mentioned in Table 1 are already activated in same-order trials, as compared to the null events. Thus, the observed areas are not newly involved in different-order trials, but instead they are involved in different- and same-order trials, but with different strength.

To identify which areas were activated overall by dual-task performance, we calculated the comparison ((different-order trials + same-order trials) $/ 2-$ null events). The results of this analysis are presented in Table 2.

\section{Discussion}

In the present study, we parametrically manipulated the demands on task-order scheduling in a PRP dual task by presenting trials with the same task order as compared to the previous trial and trials with a different task order. Specifically, this manipulation is assumed to draw on task-order scheduling demands in dual-task situations involving a capacity limited processing stage (De Jong, 1995; Luria \& Meiran, 2003). Importantly, we observed increased activation in lateral-prefrontal areas when the demands on task-order scheduling were increased. In particular, this allows refining the function of lateral-prefrontal areas in dual-task performance, thereby extending the findings of previous studies on the functional neuroanatomy of dual-task performance.

\section{Lateral prefrontal cortices}

In particular, two lateral prefrontal areas, the left posterior IFS and the right MFG, were more strongly activated in different- compared to same-order trials. We interpret this as evidence that these areas are associated with the process of task-order scheduling, i.e. the planning and monitoring of the processing order of both tasks. However, it is noteworthy to mention that these areas are highly active already during same-order trials, which indicates that task-order scheduling may be required in these trials. This is due to the fact that even if the order does not change, the short SOA between the tasks results in competition and the possibility that the tasks are processed accidentally in the wrong order. To avoid this, different authors suggested that control mechanisms monitor and, if necessary, coordinate task processing even in the case of same-order trials (Meyer \& Kieras, 1997; Norman \& Shallice, 1986). Therefore, this finding is in line with our conclusion that the presently observed areas are associated with the process of task-order scheduling.

The cortical areas along the posterior part of the IFS and MFG have been associated with a number of

Table 2 Stereotactic coordinates (Talairach \& Tournoux, 1988), anatomical locations, and $z$ scores of (local) peak activation in the comparison [(different-order + same-order) $/ 2$ - null-events]

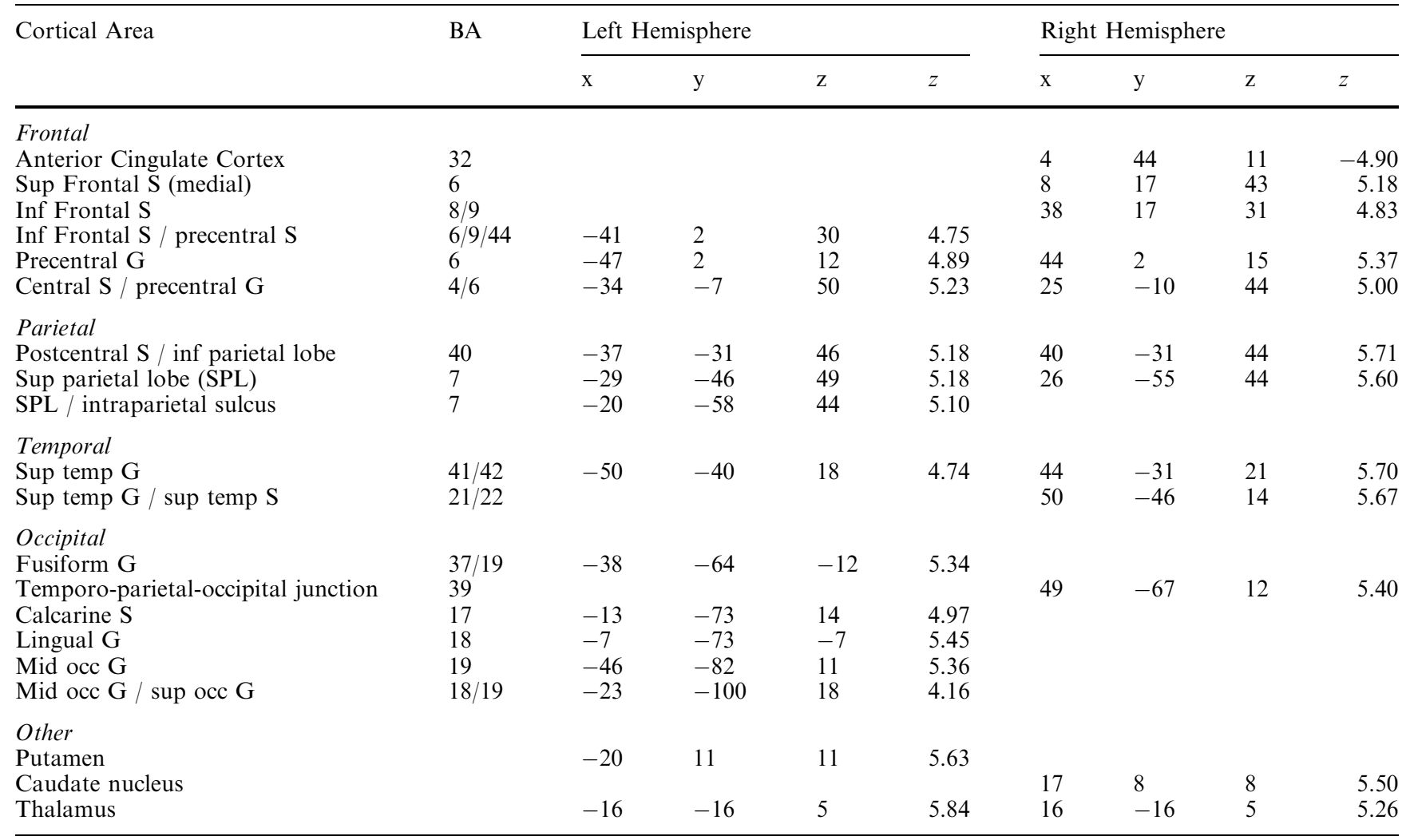

Abbreviations: BA Brodmann's area; $G$ gyrus; $S$ sulcus; Inf inferior; Sup superior; temp temporal; occ occipital 
further tasks, like the task switching paradigm (Dove, Pollmann, Schubert, Wiggins, \& von Cramon, 2000; Sohn, Ursu, Anderson, Stenger, \& Carter, 2000), the Wisconsin Card Sorting Test (WCST; Goldberg et al., 1998), or the Go-/No Go paradigm (Konishi, Nakajima, Uchida, Kikyo, Kameyama, \& Miyashita, 1999). One shared component of these paradigms is that they require the coordination of task processing under the premise that two tasks have to be performed or could be performed simultaneously and that these two tasks interfere in some way with each other. Such interference might arise by a number of causes, such as the competition for a capacity limited mechanism in the dual-task paradigm, ambiguous stimulus or response sets in the task-switching paradigm, or the need to perform a non-dominant action while a predominant action could be performed as well, as in the Go-/No Go and WCST paradigms. Thus, the converging evidence suggests that the LPFC might not only be associated with task-order scheduling in dual-task performance but in any situation requiring the coordination of different interfering tasks (Duncan, 2001; Grafman, 1995).

This idea is also consistent with assumptions of Brass and von Cramon (2004) who recently suggested that the IFJ (junction of IFS and precentral sulcus) constitutes an unique prefrontal cortical area, which is specifically associated with context-related updating of the general task representation. Such an updating process is likely to be part of the task-order scheduling mechanism, which is required particularly if the external context switches in different-order trials compared to same-order trials.

Previous research about the function of lateral prefrontal cortices suggests that the currently observed activation foci located along the posterior IFS and in the posterior MFG might subserve different functions for task control (e.g. D'Esposito et al., 1998; Owen, 2000; Smith \& Jonides, 1999). In more detail, the IFS might be related to the retrieval, implementation, and maintenance of the task set, i.e. the set of stimulusresponse associations (Brass \& von Cramon, 2004; Bunge, Kahn, Wallis, Miller, \& Wagner, 2003). Opposed to this, the posterior MFG might be related to monitoring (Petrides, Alivisatos, Meyer, \& Evans, 1993), interference resolution (Bunge, Ochsner, Desmond, Glover, \& Gabrieli, 2001), and the selection of the appropriate task (Rowe, Toni, Josephs, Frackowiak, \& Passingham, 2000). For this, the MFG might operate on and/or modulate the contents stored in the IFS (Owen, Evans, \& Petrides, 1996; Petrides, 1996). Thus, in different-order trials, the MFG might re-arrange, e.g. by activating and/or inhibiting, representations stored in the IFS. Alternatively, the MFG might monitor the presentation order of the tasks and initiate an updating of the task representations performed by the IFS. Further studies are clearly needed to dissociate the functionality of these two areas for dual-task processing.

\section{Parietal cortices}

The manipulation of task-scheduling demands resulted not only in LPFC activation but also in activation of parietal areas, e.g. along the IPS. This observation is in line with a number of studies showing that the demand to coordinate task processing is associated with a network of areas consisting mainly of prefrontal and parietal cortices. Several authors have suggested a functional dissociation between prefrontal and parietal areas for the coordination of task processing. In more detail, it has been proposed that the prefrontal cortex is associated with the initiation and coordination of attentional processes while the parietal cortex is implementing these processes (Clark, Egan, McFarlane, Morris, Weber, Sonkkilla, et al., 2000; Corbetta \& Shulman, 2002; Hopfinger, Buonocore, \& Mangun, 2000; Norman \& Shallice, 1986). In other words, it has been proposed that the top-down control of the prefrontal cortex influences the information processing in parietal association cortices (Cohen, Braver, \& O'Reilly, 1996; Desimone, 1996; Miller, 2000; Smith \& Jonides, 1999). Thus, the task scheduling organized by the prefrontal cortex might be realised by or rely on its interaction with superior parietal areas (cf. Bunge, Hazeltine, Scanlon, Rosen, \& Gabrieli, 2002). Although not completely conclusive, our data are consistent with these assumptions.

Implications for cognitive models of dual-task performance

It is generally assumed that there is a relation between fMRI activation and cognitive processing, insofar as additional or increased fMRI activation in one task compared to another is indicative of additional or increased cognitive demands in that task (Beeck, Wagemans, \& Vogels, 2001; Humphreys \& Price, 2001; Logothetis, Pauls, Augath, Trinath, \& Oeltermann, 2001; Sarter, Berntson, \& Cacioppo, 1996; cf. also Koch, Ruge, Brass, Rubin, Meiran, \& Prinz, 2003). Accordingly, the present finding of higher fMRI-activation in different-order trials indicate that these trials are associated with increased cognitive demands as compared to same-order trials. But what are the implications of this conclusion for contemporary cognitive models of dualtask performance?

Two mechanisms have been differentiated about how the tasks are processed if a capacity limited processing bottleneck requiring serial processing of the component tasks is present, passive queuing (Pashler, 1994) and active scheduling (De Jong, 1995; Luria \& Meiran, 2003; cf. also Jiang et al., 2004). Importantly, both make different predictions about the cognitive demands in same- and different-order trials. According to passive queuing, the tasks are simply processed on a first-comes first-served basis. If the bottleneck is occupied by processing the first task, the representations referring to the second task are 
held in a passive queue until the bottleneck has finished processing the first task. Thus, this account does not assume increased cognitive demands associated with the scheduling of the tasks, monitoring task performance, or, more generally, with any type of controlled/active interference resolution (see e.g. Pashler, 1994). Because this account assumes that the processing order is determined solely by the arrival times of the task processes at the capacity-limited processing stage, different-order trials should impose the same cognitive demands as same-order trials (cf. De Jong, 1995). Therefore, the passive queuing hypothesis predicts both conditions to elicit identical fMRI activation patterns.

But this is clearly not the case. Opposed to the prediction of the passive queuing account, we observed higher fMRI activation in different-order than in sameorder trials. This finding is in perfect accordance with active scheduling accounts, which assume that task processing is actively monitored and controlled. For instance, it has been proposed that task-order scheduling, stopping Task 2 processing, switching the bottleneck from Task 1 to Task 2 after processing of Task 1 has finished, and reinstating Task 2 processing are processes which require active executive control and monitoring (De Jong, 1995; Logan \& Gordon, 2001; Luria \& Meiran, 2003; Meyer \& Kieras, 1997). Active scheduling accounts are based on the interpretation of known empirical findings suggesting that task order can be prepared in advance and that, if this preparation is wrong, the re-organisation of task processing results in increased cognitive demands as compared to situations where a re-organisation is not required (De Jong, 1995; Luria \& Meiran, 2003). Accordingly, active scheduling accounts are consistent with our observation of higher fMRI activation in different-order than in same-order trials. Taken together, the current finding supports active scheduling accounts of dual-task performance. However, note that the assumption of an active scheduling mechanism in dual tasks does not contradict the assumption of a structural central bottleneck (Pashler, 1994; see also Schubert, 1999). Instead, in our view active scheduling is, in particular, necessary to organise competition of task processing at capacity limited processing stages in dual-task situations.

It has to be noted that the present findings do not allow deciphering the exact mechanism of task-order scheduling. For instance, it might be that task-order scheduling operates exclusively as a priming of the stimulus category and/or modality of the task expected to be first. However, in our view it is not plausible that task-order scheduling operates solely at the stimulus level and not at the level of tasks or task sets, i.e. at the level of stimulus-response associations. In particular, the findings of De Jong (1995) and Luria and Meiran (2003) suggest that participants not only prepare in advance the first task (or first stimulus processing) but also the specific switch of the bottleneck from task-1 processing to task-2 processing. For instance, Luria and Meiran (2003) reported an overadditive interaction between
SOA and task order onto the reaction times in the second task (RT2). Most importantly, the increase of RT2 in different-order compared to same-order trials at short compared to long SOA vanished with sufficient task preparation time. This, especially, suggests that taskorder scheduling does not exclusively operate as a mechanism that primes the first stimulus. Instead it works at the level of the whole representation of both component tasks (see Luria and Meiran, 2003, for a detailed discussion). This conclusion is additionally supported by neuroimaging studies. Recent findings of imaging studies suggest that the region of the LPFC, which was activated in the present study, is strongly associated with coordination at the task level (see Discussion section "Lateral prefrontal cortices"), while stimulus- or response-related order processing is more likely to be associated with the precentral gyrus/sulcus and/or superior parietal cortices (e.g. Coull \& Nobre, 1998; Herath, Klingberg, Young, Amunts, \& Roland, 2001; Schubotz, Friederici, \& von Cramon, 2000; see as well Schubert \& Szameitat, 2003). Taken together, we think that task-order scheduling operates, at least partly, at the level of the tasks (see also Brass \& von Cramon, 2004).

\section{Related findings}

In a recent study, Jiang et al. (2004) parametrically manipulated the temporal overlap of the tasks by comparing a short and a long SOA and proposed that dualtask related areas should be activated more strongly at short than at long SOA. However, Jiang et al. failed to find any significant difference between the activation patterns at different SOAs. Therefore, they argued in favour of the passive queuing approach. The reason for the different findings of Jiang et al. and the present study are not completely clear, but may be related to methodological issues and to differences between the tasks used by Jiang et al. and in the present study. Firstly, Jiang et al. argue on the basis of accepting the null hypothesis, which suffers from serious methodological caveats (e.g. Cortina \& Folger, 1998; Kluger \& Tikochinsky, 2001). Secondly, the task used by Jiang et al. might have been considerably easier than the task in the present study, which might explain the lack of a significant difference between the corresponding experimental conditions. For instance, Jiang et al. used 2-choice reaction tasks while we used more complex 3-choice tasks (but see results of Herath et al., 2001). Additionally, the SOA was manipulated between $48 \mathrm{~s}$ lasting blocks in the Jiang et al. study, while it was manipulated between trials in most previous PRP imaging studies (Herath et al., 2001; Schubert \& Szameitat, 2003; Szameitat et al., 2002; but see Koch, Metin, \& Schuch, 2003). Thus, in the study of Jiang et al. participants performed a dual task with the same order and same SOA throughout a block, which might have resulted in only very moderate demands on cognitive control pro- 
cesses involved in dual-task processing. We suggest that these demands might not have been sufficient to elicit stable dual-task related hemodynamic responses (see also Szameitat, 2003).

Given that in the present experiment two different tasks, one auditory and one visual, were presented intermingled, the question about the relation of our paradigm to the task-switching paradigm (Jersild, 1927) arises. Although there is indeed one, a task-switching account cannot explain the current findings for the following reason (cf. Lien \& Ruthruff, 2004). In differentorder trials, the component tasks locally repeat (e.g., visual- auditory - > auditory-visual), which means that the demands to switch between the component tasks are low. Opposed to this, in same-order trials, the tasks are locally different (visual- auditory - > visual-auditory), which should result in higher task-switching demands. Therefore, the task-switching account would predict associated cortical areas to be activated more strongly in same-order trials than in different-order trials (Dove et al., 2000). However, not a single cortical area showed this pattern. Instead, the LPFC was activated more strongly in different-order trials, which involved lower task-switching, but higher task-order scheduling demands ${ }^{3}$.

\section{Conclusion}

The ability to coordinate different interfering tasks is crucial for our everyday life, because it enables goaloriented and coherent behaviour even in situations where different potential actions interfere with or distract each other. Thus, the present findings give insight about how the brain realises complex human behaviour. We showed that areas of the LPFC are involved in the re-organisation of behaviour if current behavioural goals change rapidly (Duncan, 2001; Fuster, 2000). In more detail, the LPFC seems to enable the fast recognition of a changed action goal, as is the case in a different-order trial, and the appropriate re-organisation of task processing to accomplish this goal. Accordingly, the LPFC seems to shield from distraction to enable persistent and coherent behaviour while at the same time enabling an unique flexibility of behaviour if the action goal changes (Duncan, 2001; Miller \& Cohen, 2001).

\footnotetext{
${ }^{3}$ Additionally, one might argue that the order of the component tasks was confounded with hand order. In other words, in differentorder trials, not only the order of the component tasks changed, but also the order in which the hands had to be used to give the responses. However, it seems unlikely that this confound can explain the current data. Firstly, in Experiment 3 of Luria and Meiran (2003) hand order was kept constant, and still the same behavioural differences between different-order and same-order trials were observed. Secondly, previous imaging research suggests that bimanual coordination and sequencing is mainly associated with premotor areas of the precentral gyrus, but not in the prefrontal areas observed in the current study (see e.g. Debaere, Wenderoth, Sunaert, Van Hecke, \& Swinnen, 2004; Koeneke, Lutz, Wüstenberg, \& Jäncke, 2004).
}

In summary, we showed that cortical areas of the posterior LPFC along the IFS and in the MFG are involved in the co-ordination and re-organisation of the processing of two concurrent tasks, which interfere with each other due to a limited processing mechanism. The present approach and findings allow a much more detailed description of the functionality of the LPFC for dual-task performance as compared to previous studies. We conclude that the LPFC is essential for the organisation of complex human behaviour especially in situations where contradictory or interfering information has to be processed in order to execute goal oriented behaviour.

Acknowledgements This experiment is part of Andre Szameitat's doctoral dissertation, which was conducted at the Max Planck Institute for Human Cognitive and Brain Sciences, Leipzig, and supervised by Torsten Schubert. We thank Anke Mempel, Mandy Naumann, Anke Pitzmaus, and Kathrin Wiesner for their assistance in data acquisition, Philip Dean for proof-reading the English manuscript, and Silvia Bunge, Roy Luria, and Iring Koch for their valuable comments on an earlier version of the manuscript.

\section{References}

Beeck, H.o.d., Wagemans, J., \& Vogels, R. (2001). Can neuroimaging really tell us what the human brain is doing? The relevance of indirect measures of population activity. Acta Psychologica, Amsterdam, 107(1-3), 323-351.

Brass, M., \& von Cramon, D.Y. (2004). Decomposing components of task preparation with functional magnetic resonance imaging. Journal of Cognitive Neuroscience, 16(4), 609-620.

Braun, J. (1998). Divided attention: Narrowing the gap between brain and behavior. In: Parasuraman, R. (ed), The attentive brain. (pp. 327-351). Cambridge, MA, US: The MIT Press.

Braver, T.S., Cohen, J.D., Nystrom, L.E., Jonides, J., Smith, E.E., \& Noll, D.C. (1997). A parametric study of prefrontal cortex involvement in human working memory. Neuroimage, 5(1), 4962.

Bunge, S.A., Hazeltine, E., Scanlon, M.D., Rosen, A.C., \& Gabrieli, J.D. (2002). Dissociable contributions of prefrontal and parietal cortices to response selection. Neuroimage, 17(3), 15621571.

Bunge, S.A., Kahn, I., Wallis, J.D., Miller, E.K., \& Wagner, A.D. (2003). Neural circuits subserving the retrieval and maintenance of abstract rules. Journal of Neurophysiology, 90(5), 3419-3428.

Bunge, S.A., Ochsner, K.N., Desmond, J.E., Glover, G.H., \& Gabrieli, J.D. (2001). Prefrontal regions involved in keeping information in and out of mind. Brain, 124(Pt 10), 2074-2086.

Burock, M.A., Buckner, R.L., Woldorff, M.G., Rosen, B.R., \& Dale, A.M. (1998). Randomized event-related experimental designs allow for extremely rapid presentation rates using functional MRI. Neuroreport, 9(16), 3735-3739.

Clark, C.R., Egan, G.F., McFarlane, A.C., Morris, P., Weber, D., Sonkkilla, C., et al. (2000). Updating working memory for words: A PET activation study. Human Brain Mapping, 9(1), 42-54.

Cohen, J.D., Braver, T.S., \& O'Reilly, R.C. (1996). A computational approach to prefrontal cortex, cognitive control and schizophrenia: Recent developments and current challenges. Philosophical Transactions of the Royal Society of London. Series B: Biological Sciences, 351(1346), 1515-1527.

Corbetta, M., \& Shulman, G.L. (2002). Control of goal-directed and stimulus-driven attention in the brain. Nature Reviews Neuroscience, 3(3), 201-215.

Cortina, J.M., \& Folger, R.G. (1998). When is it acceptable to accept a null hypothesis: No way, Jose? Organizational Research Methods, 1(3), 334-350. 
Coull, J.T., \& Nobre, A.C. (1998). Where and when to pay attention: The neural systems for directing attention to spatial locations and to time intervals as revealed by both PET and fMRI. The Journal of Neuroscience, 18(18), 74267435.

De Jong, R. (1995). The role of preparation in overlapping-task performance. The Quarterly Journal of Experimental Psychology. A, Human Experimental Psychology, 48(1), 2-25.

Debaere, F., Wenderoth, N., Sunaert, S., Van Hecke, P., \& Swinnen, S.P. (2004). Cerebellar and premotor function in bimanual coordination: parametric neural responses to spatiotemporal complexity and cycling frequency. Neuroimage, 21(4), 14161427.

Desimone, R. (1996). Neural mechanisms for visual memory and their role in attention. Proceedings of the National Academy of Sciences of the United States of America, 93(24), 1349413499.

D'Esposito, M., Aguirre, G.K., Zarahn, E., Ballard, D., Shin, R.K., \& Lease, J. (1998). Functional MRI studies of spatial and nonspatial working memory. Brain Research. Cognitive Brain Research, 7(1), 1-13.

D'Esposito, M., Detre, J.A., Alsop, D.C., Shin, R.K., Atlas, S., \& Grossman, M. (1995). The neural basis of the central executive system of working memory. Nature, 378(6554), 279-281.

Dove, A., Pollmann, S., Schubert, T., Wiggins, C.J., \& von Cramon, D.Y. (2000). Prefrontal cortex activation in task switching: An event-related fMRI study. Brain Research. Cognitive Brain Research, 9(1), 103-109.

Duncan, J. (1979). Divided attention: The whole is more than the sum of its parts. Dual-task interference as an indicator of on-line programming in simple movement sequences. Journal of Experimental Psychology: Human Perception and Performance, 5(2), 216-228.

Duncan, J. (2001). An adaptive coding model of neural function in prefrontal cortex. Nature Reviews Neuroscience, 2(11), 820829.

Friston, K.J., Holmes, A.P., Worsley, K.J., Poline, J.-P., Frith, C.D., \& Frackowiak, R.S.J. (1995). Statistical Parametric Maps in Functional Imaging: A General Linear Approach. Human Brain Mapping, 2, 189-210.

Friston, K.J., Price, C.J., Fletcher, P., Moore, C., Frackowiak, R.S., \& Dolan, R.J. (1996). The trouble with cognitive subtraction. Neuroimage, 4(2), 97-104.

Friston, K.J., Zarahn, E., Josephs, O., Henson, R.N., \& Dale, A.M. (1999). Stochastic designs in event-related fMRI. Neuroimage, 10(5), 607-619.

Fuster, J.M. (2000). Executive frontal functions. Experimental Brain Research, 133(1), 66-70.

Goldberg, T.E., Berman, K.F., Fleming, K., Ostrem, J., Horn, J.D.V., Esposito, G., et al. (1998). Uncoupling Cognitive Workload and Prefrontal Cortical Physiology: A PET rCBF Study. Neuroimage, 7(4), 296-303.

Grafman, J. (1995). Similarities and distinctions among current models of prefrontal cortical functions. Annals of the New York Academy of Sciences, 769, 337-368.

Herath, P., Klingberg, T., Young, J., Amunts, K., \& Roland, P. (2001). Neural correlates of dual task interference can be dissociated from those of divided attention: An fMRI study. Cerebral Cortex, 11(9), 796-805.

Hopfinger, J.B., Buonocore, M.H., \& Mangun, G.R. (2000). The neural mechanisms of top-down attentional control. Nature Neuroscience, 3(3), 284-291.

Humphreys, G.W., \& Price, C.J. (2001). Cognitive neuropsychology and functional brain imaging: Implications for functional and anatomical models of cognition. Acta Psychologica, 107(13), 119-153.

Jersild, A.T. (1927). Mental set and shift. Archives of Psychology, 14(Whole No. 89).

Jiang, Y., Saxe, R., \& Kanwisher, N. (2004). Functional magnetic resonance imaging provides new constraints on theories of the psychological refractory period. Psychological Science, 15(6), 390-396.
Josephs, O., \& Henson, R.N. (1999). Event-related functional magnetic resonance imaging: Modelling, inference and optimization. Philosophical Transactions of the Royal Society of London. Series B: Biological Sciences, 354(1387), 1215-1228.

Josephs, O., Turner, R., \& Friston, K.J. (1997). Event-Related fMRI. Human Brain Mapping, 5, 243-248.

Kluger, A.N., \& Tikochinsky, J. (2001). The error of accepting the "theoretical" null hypothesis: The rise, fall, and resurrection of commonsense hypotheses in psychology. Psychological Bulletin, 127(3), 408-423.

Koch, I., Metin, B., \& Schuch, S. (2003). The role of temporal unpredictability for process interference and code overlap in perception-action dual tasks. Psychological Research, 67(4), 244-252.

Koch, I., Ruge, H., Brass, M., Rubin, O., Meiran, N., \& Prinz, W. (2003). Equivalence of cognitive processes in brain imaging and behavioral studies: evidence from task switching. Neuroimage, 20(1), 572-577.

Koeneke, S., Lutz, K., Wüstenberg, T., \& Jäncke, L. (2004). Bimanual versus unimanual coordination: what makes the difference? Neuroimage, 22(3), 1336-1350.

Konishi, S., Nakajima, K., Uchida, I., Kikyo, H., Kameyama, M., \& Miyashita, Y. (1999). Common inhibitory mechanism in human inferior prefrontal cortex revealed by event-related functional MRI. Brain, 122(Pt 5), 981-991.

Lien, M.C., \& Ruthruff, E. (2004). Task switching in a hierarchical task structure: evidence for the fragility of the task repetition benefit. Journal of Experimental Psychology. Learning, Memory, and Cognition, 30(3), 697-713.

Logan, G.D., \& Gordon, R.D. (2001). Executive control of visual attention in dual-task situations. Psychological Review, 108(2), 393-434.

Logothetis, N.K., Pauls, J., Augath, M., Trinath, T., \& Oeltermann, A. (2001). Neurophysiological investigation of the basis of the fMRI signal. Nature, 412(6843), 150-157.

Lohmann, G., Müller, K., Bosch, V., Mentzel, H., Hessler, S., Chen, L., et al. (2001). Lipsia - A new software system for the evaluation of functional magnetic resonance images of the human brain. Computerized Medical Imaging and Graphics, 25(6), 449-457.

Luria, R., \& Meiran, N. (2003). Online order control in the psychological refractory period paradigm. Journal of Experimental Psychology: Human Perception and Performance, 29(3), 556574.

Meyer, D.E., \& Kieras, D.E. (1997). A computational theory of executive cognitive processes and multiple-task performance: Part 1. Basic mechanisms. Psychological Review, 104(1), 3-65.

Miezin, F.M., Maccotta, L., Ollinger, J.M., Petersen, S.E., \& Buckner, R.L. (2000). Characterizing the hemodynamic response: Effects of presentation rate, sampling procedure, and the possibility of ordering brain activity based on relative timing. Neuroimage, 11(6 Pt 1), 735-759.

Miller, E.K. (2000). The prefrontal cortex and cognitive control. Nature Reviews Neuroscience, 1(1), 59-65.

Miller, E.K., \& Cohen, J.D. (2001). An integrative theory of prefrontal cortex function. Annual Review of Neuroscience, 24, 167-202.

Norman, D.A., \& Shallice, T. (1986). Attention to action: Willed and automatic control of behavior. In: Davidson, R.J., Schwartz, G.E., \& Shapiro, D. (eds.), Consciousness and self-regulation: Advances in research and theory (Vol. 4, pp. 1-18). New York: Plenum Press.

Oldfield, R.C. (1971). The assessment and analysis of handedness: The Edinburgh Inventory. Neuropsychologia, 9, 97-113.

Owen, A.M. (2000). The role of the lateral frontal cortex in mnemonic processing: The contribution of functional neuroimaging. Experimental Brain Research, 133(1), 33-43.

Owen, A.M., Evans, A.C., \& Petrides, M. (1996). Evidence for a two-stage model of spatial working memory processing within the lateral frontal cortex: A positron emission tomography study. Cerebral Cortex, 6(1), 31-38. 
Pashler, H. (1994). Dual-task interference in simple tasks: Data and theory. Psychological Bulletin, 116(2), 220-244.

Petrides, M. (1996). Specialized systems for the processing of mnemonic information within the primate frontal cortex. Philosophical Transactions of the Royal Society of London. Series B: Biological Sciences, 351(1346), 1455-1461; discussion 1461-1452.

Petrides, M., Alivisatos, B., Meyer, E., \& Evans, A.C. (1993). Functional activation of the human frontal cortex during the performance of verbal working memory tasks. Proceedings of the National Academy of Sciences of the United States of America, 90(3), 878-882.

Rosen, B.R., Buckner, R.L., \& Dale, A.M. (1998). Event-related functional MRI: Past, present, and future. Proceedings of the National Academy of Sciences of the United States of America, 95(3), 773-780.

Rowe, J.B., Toni, I., Josephs, O., Frackowiak, R.S., \& Passingham, R.E. (2000). The prefrontal cortex: Response selection or maintenance within working memory? Science, 288(5471), $1656-1660$.

Sarter, M., Berntson, G.G., \& Cacioppo, J.T. (1996). Brain imaging and cognitive neuroscience. Toward strong inference in attributing function to structure. The American Psychologist, $51(1), 13-21$.

Schubert, T. (1999). Processing differences between simple and choice reactions affect bottleneck localization in overlapping tasks. Journal of Experimental Psychology: Human Perception and Performance, 25(2), 408-425.

Schubert, T., \& Szameitat, A.J. (2003). Functional neuroanatomy of interference in overlapping dual tasks: an fMRI study. Brain Research. Cognitive Brain Research, 17(3), 733746.

Schubotz, R.I., Friederici, A.D., \& von Cramon, D.Y. (2000). Time perception and motor timing: A common cortical and subcortical basis revealed by fMRI. Neuroimage, 11(1), 1-12.

Smith, E.E., \& Jonides, J. (1999). Storage and executive processes in the frontal lobes. Science, 283(5408), 1657-1661.

Sohn, M.H., Ursu, S., Anderson, J.R., Stenger, V.A., \& Carter, C.S. (2000). The role of prefrontal cortex and posterior parietal cortex in task switching. Proceedings of the National Academy of Sciences of the United States of America, 97(24), 1344813453.

Szameitat, A.J. (2003). The functionality of the lateral prefrontal cortex for dual-task performance [German]. Leipzig: Max Planck Institute of Cognitive Neuroscience, MPI Series in Cognitive Neuroscience (32).

Szameitat, A.J., Schubert, T., Müller, K., \& von Cramon, D.Y. (2002). Localization of executive functions in dual-task performance with fMRI. Journal of Cognitive Neuroscience, 14(8), 1184-1199.

Talairach, P., \& Tournoux, J. (1988). A Stereotactic Coplanar Atlas of the Human Brain. Stuttgart: Thieme.

Thirion, J.P. (1998). Image matching as a diffusion process: An analogy with Maxwell's demons. Medical Image Analysis, 2(3), 243-260.

Umiltà, C., Nicoletti, R., Simion, F., Tagliabue, M.E., \& Bagnara, S. (1992). The cost of a strategy. European Journal of Cognitive Psychology, 4(1), 21-40. 